\title{
Parametrization of Algebraic Points of Low Degrees on the Schaeffer Curve
}

\author{
Moussa Fall ${ }^{1}$ \\ ${ }^{1}$ Department of Mathematics, Faculty of Science and Technology University, Assane Seck, Ziguinchor
}

\section{Article Info}

Keywords: Degree of algebraic points, Plan curve, Rational points.

2010 AMS: 11D68, 12FO5, 14H40, $14 \mathrm{H} 50$

Received: 2 May 2021

Accepted: 19 August 2021

Available online: 31 August 2021

\begin{abstract}
In this paper, we give a parametrization of algebraic points of degree at most 4 over $\mathbb{Q}$ on the schaeffer curve $\mathscr{C}$ of affine equation : $y^{2}=x^{5}+1$. The result extends our previous result which describes in [5] ( Afr. Mat 29:1151-1157, 2018) the set of algebraic points of degree at most 3 over $\mathbb{Q}$ on this curve.
\end{abstract}

\section{Introduction}

Let $\mathscr{C}$ be a smooth projective plane curve defined over $K$. For all algebraic extension field $K$ of $\mathbb{Q}$, we denote by $\mathscr{C}(K)$ the set of $K$-rational points of $\mathscr{C}$ over $K$ and $\mathscr{C}^{(d)}(\mathbb{Q})$ the set of algebraic points of $\leq d$ over $\mathbb{Q}$. The degree of an algebraic point $R$ is the degree of its field of definition on $\mathbb{Q}: \operatorname{deg}(R)=[\mathbb{Q}(R): \mathbb{Q}]$.

A famous theorem of Fatling show that if $\mathscr{C}$ is a smooth projective plane curve defined over $K$ of genus $g \geq 2$, then $\mathscr{C}(K)$ is finite. Fatling's proof is still ineffective in the sense that it does not provide an algorithm for computing $\mathscr{C}(K)$. A most precise theorem of Debarre and Klasen [4] show that if $\mathscr{C}$ be a smooth projective plane curve defined by an equation of degree $d \geq 7$ with rational coefficients then $\mathscr{C}^{(d-2)}(\mathbb{Q})$ is finite. This theorem often us to characterize the set $\mathscr{C}^{(2)}(\mathbb{Q})$ of all algebraic points of degree $\leq 2$ over $\mathbb{Q}$.

Currently for curve $\mathscr{C}$ defined over a numbers field $K$ of genus $g \geq 2$, there is no known algorithm for computing the set $\mathscr{C}(K)$ or for deciding if $\mathscr{C}(K)$ is empty. But there is a bag of strikes that can be used to show that $\mathscr{C}(K)$ is empty, or to determine $\mathscr{C}(K)$ if it is not empty. Among these methods, we can cite the local method, Chabauty method [2], Descent method [7], mordell-weil sieves method [1]. These methods often succeed with less than full knowledge of the jacobian $J(\mathbb{Q})$ of the curve . If $J(\mathbb{Q})$ is finite then it is no hard to determine $\mathscr{C}(\mathbb{Q})$ and to generalize for all number field $K$.

Previous works ([3] and [5]) have studied the algebraic points of degree at most 3 on the schaeffer curve of affine equation $y^{2}=x^{5}+1$ denoted $\mathscr{C}$. The curve $\mathscr{C}$ is hyperelliptic of genus $g=2$ and of rank null by [3].

Let's denote $P_{0}=(-1,0), P_{1}=(0,1), \overline{P_{1}}=(0,-1), Q_{1}=(1+i, 1-2 i), Q_{2}=(1-i, 1+2 i), \overline{Q_{1}}=(1+i,-1+2 i), \overline{Q_{2}}=(1-i,-1-2 i)$ and $\infty$ the point at infinity.

The purpose of this note is to determine the algebraic parametrization of all algebraic points of degree at most four on the curve $\mathscr{C}_{s}$ over the rationnal numbers field $\mathbb{Q}$ using ideas in [5] (Afr. Mat 29:1151-1157, 2018).

\section{Auxiliary results}

Lemma 2.1. Let $x$ and $y$ be the rational functions defined on $\mathscr{C}_{s}$ by $x(X, Y, Z)=\frac{X}{Z}$ and $y(X, Y, Z)=\frac{Y}{Z}$ :

- $\operatorname{div}(y-1)=5 P_{1}-5 \infty ; \quad \operatorname{div}(y+1)=5 \bar{P}_{1}-5 \infty ;$

- $\operatorname{div}(x)=P_{1}+\bar{P}_{1}-2 \infty ; \quad \operatorname{div}(x+1)=2 P_{0}-2 \infty$

- $\operatorname{div}(y)=A_{0}+A_{1}+A_{2}+A_{3}+A_{4}-5 \infty$ where $A_{i}=\exp \left(i(2 k+1) \frac{\pi}{5}\right)$. 
Denote by $\mathscr{L}(m \infty)$ the $\overline{\mathbb{Q}}$-vector space of rational functions defined by $\mathscr{L}(m \infty)=\left\{f \in \overline{\mathbb{Q}}\left(\mathscr{C}_{s}\right)^{*} \mid \operatorname{div}(f) \geq-m \infty\right\} \cup\{0\}$ :

- $\mathscr{L}(\infty)=\langle 1\rangle$

- $\mathscr{L}(2 \infty)=\mathscr{L}(3 \infty)=\langle 1, x\rangle$

- $\mathscr{L}(4 \infty)=\left\langle 1, x, x^{2}\right\rangle$

- $\mathscr{L}(5 \infty)=\left\langle 1, x, x^{2}, y\right\rangle$

- $\mathscr{L}(6 \infty)=\left\langle 1, x, x^{2}, y, x^{3}\right\rangle$

Proof. See [5]

Lemma 2.2. We consider the divisor $D$ on the curve $\mathscr{C}_{S}$ :

- $D=[(-1,0)+(0,1)-2 \infty]=\left[P_{0}+P_{1}-2 \infty\right]$

- $2 D=[2(0,1)-2 \infty]=\left[2 P_{1}-2 \infty\right]$

- $3 D=[(1+i, 1-2 i)+(1-i, 1+2 i)-2 \infty]=\left[Q_{1}+Q_{2}-2 \infty\right]$

- $4 D=[(0,-1)-\infty]=\left[\bar{P}_{1}-\infty\right]$

- $5 D=[(-1,0)-\infty]=\left[P_{0}-\infty\right]$

- $6 D=[(0,1)-\infty]=\left[P_{1}-\infty\right]$

- $7 D=[(1+i,-1+2 i)+(1-i,-1-2 i)-2 \infty]=\left[\bar{Q}_{1}+\bar{Q}_{2}-2 \infty\right]$

- $8 D=[2(0,-1)-2 \infty]=\left[2 \bar{P}_{1}-2 \infty\right]$

- $9 D=[(-1,0)+(0,-1)-2 \infty]=\left[P_{0}+\bar{P}_{1}-2 \infty\right]$

- $10 D=0$.

The Mordell-weil groupe of the curve $\mathscr{C}_{s}$ is $J(\mathbb{Q}) \cong(\mathbb{Z} / 10 \mathbb{Z}) \cong\langle D\rangle=\{m D \mid 0 \leq m \leq 9\}$.

Proof. See [3].

\section{Main result}

Our main result is the following theorem

Theorem 3.1. The algebraic points of degree 4 over $\mathbb{Q}$ on the curve $\mathscr{C}_{s}$ are given by the union of the following sets : $\mathscr{G}_{0} \cup \mathscr{G}_{1} \cup \mathscr{G}_{2} \cup \mathscr{G}_{3} \cup \mathscr{G}_{4} \cup \mathscr{G}_{5}$ with

- $\left.\mathscr{G}_{0}=\left\{\left(x, \pm \sqrt{x^{2}+1}\right)\right) \mid[\mathbb{Q}(x): \mathbb{Q}]=2, x^{2}-2 x+2 \neq 0\right\}$;

- $\mathscr{G}_{1}=\left\{\begin{array}{l}\left(x, \pm\left(-1+(-1-a+c) x-a x^{2}-c x^{3}\right) \mid a, c \in \mathbb{Q}, c \neq 0 \text { et } a \neq c-1, x \text { root of }\right. \\ B_{1}(x)=c^{2} x^{4}+\left(2 a c-c^{2}-1\right) x^{3}+\left(a^{2}-c^{2}+2 c+1\right) x^{2}+\left(a^{2}+2 a-2 a c+c^{2}-1\right) x+2 a-2 c+2=0\end{array}\right\} ;$

- $\mathscr{G}_{2}=\left\{\left(x, \pm\left(c x^{3}+a x^{2}-1\right)\right) \mid a, c \in \mathbb{Q}^{*}, a \neq c+1, x\right.$ root of $\left.B_{2}(x)=c^{2} x^{4}+2 a c x^{3}-x^{3}+a^{2} x^{2}-2 c x-2 a=0\right\}$;

- $\mathscr{G}_{3}=\left\{\begin{array}{l}\left(x, \pm\left(-3-2 a-4 c+(2+2 a+2 c) x-a x^{2}-c x^{3}\right)\right) \mid a, c \in \mathbb{Q}, a \neq-1-2 c, c \neq 0, x \text { root of } B_{3}(x)=c^{2} x^{4}+ \\ \left(2 c^{2}+2 a c-1\right) x^{3}+\left(-2 c^{2}-4 c+a^{2}-2\right) x^{2}+\left(-4 a c-2 c-2 a^{2}-4 a-2\right) x+8 c^{2}+8 a c+12 c+2 a^{2}+6 a+4\end{array}\right\} ;$

- $\mathscr{G}_{4}=\left\{\left(x, \pm\left(1+a x+c x^{2}\right)\right) \mid a, c \in \mathbb{Q}, a \neq 0, x\right.$ root of $\left.B_{4}(x)=-x^{4}+c^{2} x^{3}+2 a c x^{2}+\left(2 c+a^{2}\right) x+2\right\}$;

- $\mathscr{G}_{5}=\left\{\begin{array}{l}\left(x, \pm\left(-a+(-a-c) x-c x^{2}\right)\right) \mid, a, c \in \mathbb{Q}, a \neq \pm 1, x \text { root of } B_{5}(x)=-x^{4}+\left(c^{2}+1\right) x^{3}+\left(c^{2}+2 a c-1\right) x^{2} \\ +\left(2 a c+a^{2}+1\right) x+a^{2}-1\end{array}\right\}$.

Proof of theoreme.

Let $R \in \mathscr{C}_{S}(\overline{\mathbb{Q}})$ with $[\mathbb{Q}(R): \mathbb{Q}]=4$. Let $R_{1}, R_{2}, R_{3}, R_{4}$ be the Galois conjugates of $R$. We have

$$
\left[R_{1}+R_{2}+R_{3}+R_{4}-4 \infty\right] \in J(\mathbb{Q})
$$

from lemma (2.2), we get

$$
\left[R_{1}+R_{2}+R_{3}+R_{4}-4 \infty\right]=m D, \quad 0 \leq m \leq 9
$$

Now for any integer $m$ such that $0 \leq m \leq 9$, we have $m D=(m-10) D$, so

$$
\left[R_{1}+R_{2}+R_{3}+R_{4}-4 \infty\right]=(m-10) D, \quad 0 \leq m \leq 9 .
$$

Our proof is divided in five cases

\section{$\underline{\text { Case } m=0}$}

Formula $(\star)$ becomes

$$
\left[R_{1}+R_{2}+R_{3}+R_{4}-4 \infty\right]=0 .
$$

The Abel Jacobi theorem involves the existence of a function $F$ such that

$$
\operatorname{div}(F)=R_{1}+R_{2}+R_{3}+R_{4}-4 \infty
$$

so $F \in \mathscr{L}(4 \infty)$, and lemma $(2.1)$ gives $F(x, y)=a+b x+c x^{2} ; x$ must be in the $\overline{\mathbb{Q}}$ such as $[\mathbb{Q}(x): \mathbb{Q}]=2$ and $x^{2}-2 x+2 \neq 0$. We get a family of quartic points

$$
\left.\mathscr{G}_{0}=\left\{\left(x, \pm \sqrt{x^{5}+1}\right)\right) \mid x \in[\mathbb{Q}(x): \mathbb{Q}]=2, x^{2}-2 x+2 \neq 0\right\} .
$$

Cases $m=1$ and $m=9$ 
For $m=1$ : The formula $(\star)$ and lemma (2.2) give

$$
\left[R_{1}+R_{2}+R_{3}+R_{4}-4 \infty\right]=-9 D=-\left[P_{0}+\bar{P}_{1}-2 \infty\right] .
$$

This means

$$
\left[R_{1}+R_{2}+R_{3}+R_{4}+P_{0}+\bar{P}_{1}-6 \infty\right]=0
$$

The Abel Jacobi theorem involves the existence of a function $F$ such that

$$
\operatorname{div}(F)=R_{1}+R_{2}+R_{3}+R_{4}+P_{0}+\bar{P}_{1}-6 \infty .
$$

So $F \in \mathscr{L}(6 \infty)$, then $F(x, y)=u+v x+w x^{2}+d x^{3}+e y(e \neq 0)$. We have $F\left(\bar{P}_{1}\right)=F\left(P_{0}\right)=0$, so $u-e=0$ and $u-v+w-d=0$, thus

$$
F(x, y)=u+(u+w-d) x+w x^{2}+d x^{3}+u y \quad u \neq 0 .
$$

At the points $R_{i}$, we have $y=-1+(-1-a+c) x-a x^{2}-c x^{3}$ with $a=\frac{w}{u}$ and $c=\frac{d}{u}$. By substituting $y$ in $y^{2}-x^{5}-1=0$ and simplifying by $x(x+1)$ we obtain

$$
B_{1}(x)=c^{2} x^{4}+\left(2 a c-c^{2}-1\right) x^{3}+\left(a^{2}-c^{2}+2 c+1\right) x^{2}+\left(a^{2}+(2-2 c) a+c^{2}-1\right) x+2 a-2 c+2=0
$$

We must have $B_{1}(0) \neq 0$ and $B_{1}(-1) \neq 0$ which involves $a \neq c-1$ and $c \neq 0$. We have a family of quartic points

$$
\mathscr{G}_{1,1}=\left\{\left(x,+\left(-1+(-1-a+c) x-a x^{2}-c x^{3}\right)\right) \mid a, c \in \mathbb{Q}, a \neq c-1, c \neq 0, x \text { root of } B_{1}(x)=0\right\}
$$

For $m=9$ : The formula $(\star)$ and lemma (2.2) give

$$
\left[R_{1}+R_{2}+R_{3}+R_{4}-4 \infty\right]=-D=-\left[P_{0}+P_{1}-2 \infty\right]
$$

This means

$$
\left[R_{1}+R_{2}+R_{3}+R_{4}+P_{0}+P_{1}-6 \infty\right]=0
$$

The Abel Jacobi theorem involves the existence of a function $F$ such that

$$
\operatorname{div}(F)=R_{1}+R_{2}+R_{3}+R_{4}+P_{0}+P_{1}-6 \infty .
$$

So $F \in \mathscr{L}(6 \infty)$, hence $F(x, y)=u+v x+w x^{2}+d x^{3}+e y(e \neq 0)$. We have $F\left(P_{1}\right)=F\left(P_{0}\right)=0$, so $u-e=0$ et $u-v+w-d=0$, then

$$
F(x, y)=u+(u+w-d) x+w x^{2}+d x^{3}+u y \quad u \neq 0 .
$$

At the points $R_{i}$, we have $y=1+(1+a-c) x+a x^{2}+c x^{3}$ with $a=-\frac{w}{u}$ and $c=-\frac{d}{u}$. By substituting $y$ in $y^{2}-x^{5}-1=0$ and simplifying by $x(x+1)$, we have

$$
B_{1}(x)=c^{2} x^{4}+\left(2 a c-c^{2}-1\right) x^{3}+\left(a^{2}-c^{2}+2 c+1\right) x^{2}+\left(a^{2}+(2-2 c) a+c^{2}-1\right) x+2 a-2 c+2=0
$$

We must have $B_{1}(0) \neq 0$ and $B_{1}(-1) \neq 0$ involving $a \neq c-1$ and $c \neq 0$. We get a family of quartic points

$$
\mathscr{G}_{1,2}=\left\{\left(x,-\left(-1+(-1-a+c) x-a x^{2}-c x^{3}\right)\right) \mid a, c \in \mathbb{Q}, a \neq c-1, c \neq 0, x \operatorname{root} \text { of } B_{1}(x)=0\right\} .
$$

Finally, we get a second family of quartic points $\mathscr{G}_{1}=\mathscr{G}_{1,1} \cup \mathscr{G}_{1,2}$.

\section{$\underline{\text { Cases } m=2 \text { and } m=8}$}

For $m=2:$ the formula $(\star)$ becomes

This means

$$
\left[R_{1}+R_{2}+R_{3}+R_{4}-4 \infty\right]=-8 D=-\left[2 \overline{P_{1}}-2 \infty\right]
$$

$$
\left[R_{1}+R_{2}+R_{3}+R_{4}+2 \overline{P_{1}}-6 \infty\right]=0
$$

The Abel Jacobi theorem involves the existence of a function $F$ such that

$$
\operatorname{div}(F)=R_{1}+R_{2}+R_{3}+R_{4}+2 \overline{P_{1}}-6 \infty
$$

So $F \in \mathscr{L}(6 \infty)$, hence $F(x, y)=a+b x+c x^{2}+d x^{3}+e y(e \neq 0)$. The point $\bar{P}_{1}$ is order 2 , so $u-e=0$ and $v=0$, thus

$$
F(x, y)=u+w x^{2}+d x^{3}+u y
$$

At the points $R_{i}$, we have $-u y=u+w x^{2}+d x^{3}(u \neq 0)$, so $y=-1+a x^{2}+c x^{3}$ with $a=-\frac{w}{u}$ and $k=-\frac{d}{u}$. Substuting $y$ to $y^{2}=x^{5}+1$, we have

Simplifying by $x^{2}$, we have

$$
x^{2}\left(a^{2} x^{4}+2 a c x^{3}-x^{3}+a^{2} x^{2}-2 c x-2 a\right)=0 .
$$

$$
B_{2}(x)=c^{2} x^{4}+2 a c x^{3}-x^{3}+a^{2} x^{2}-2 c x-2 a .
$$

We must have $a c \neq 0$ and $a \neq c+1$. We obtain a family of quartic points :

$$
\mathscr{G}_{2,1}=\left\{\left(x,\left(c x^{3}+a x^{2}-1\right)\right) \mid a, c \in \mathbb{Q}^{*}, a \neq c+1, x \operatorname{root} \text { of } B_{2}(x)=0\right\} .
$$

For $m=8$ : by a similar argument as in case $m=2$, we have

$$
\mathscr{G}_{2,2}=\left\{\left(x,-\left(c x^{3}+a x^{2}-1\right)\right) \mid a, c \in \mathbb{Q}^{*}, a \neq c+1, x \text { root of } B_{2}(x)=0\right\} .
$$

Finally, we have the third family $\mathscr{G}_{2}=\mathscr{G}_{2,1} \cup \mathscr{G}_{2,2}$. 


\section{Cases $m=3$ and $m=7$}

For $m=3:$ the formula $(\star)$ becomes

$$
\left[R_{1}+R_{2}+R_{3}+R_{4}-4 \infty\right]=-7 D=-\left[\overline{Q_{1}}+\overline{Q_{2}}-2 \infty\right]
$$

This means

$$
\left[R_{1}+R_{2}+R_{3}+R_{4}+\overline{Q_{1}}+\overline{Q_{2}}-6 \infty\right]=0
$$

The Abel Jacobi theorem involves the existence of a function $F$ such that

$$
\operatorname{div}(F)=R_{1}+R_{2}+R_{3}+R_{4}+\overline{Q_{1}}+\overline{Q_{2}}-6 \infty .
$$

Then $F(x, y)=u+v x+w x^{2}+d x^{3}+e y(e \neq 0)$. We have $F\left(\bar{Q}_{1}\right)=F\left(\bar{Q}_{2}\right)=0$, so $u+v-2 d-e=0$ and $v+2 w+2 d+2 e=0$, hence

$$
F(x, y)=2 w+4 d+3 e+(-2 w-2 d-2 e) x+w x^{2}+d x^{3}+e y(e \neq 0) .
$$

At points $R_{i}$, we have $y=(-3-2 a-4 c)+(2+2 a+2 c) x-a x^{2}-c x^{3}$ with $a=\frac{w}{e}$ and $c=\frac{d}{e}$. Substuting $y$ into $y^{2}=x^{5}+1$ and simplifying by $x^{2}-2 x+2$, we have

$$
B_{3}(x)=c^{2} x^{4}+\left(2 c^{2}+2 a c-1\right) x^{3}+\left(-2 c^{2}-4 c+a^{2}-2\right) x^{2}+\left((-4 a-2) c-2 a^{2}-4 a-2\right) x+8 c^{2}+(8 a+12) c+2 a^{2}+6 a+4=0 .
$$

We must have $c \neq 0$ and $a \neq-1-2 c$. We get a family of quartic points

$$
\mathscr{G}_{3,1}=\left\{\left(x,\left(-3-2 a-4 c+(2+2 a+2 c) x-a x^{2}-c x^{3}\right)\right) \mid a, c \in \mathbb{Q} c \neq 0, a \neq-1-2 c, x \text { root of } B_{3}(x)=0\right\}
$$

For $m=7$ : by a similar argument as in previous case, we get a family of quartic points

$$
\mathscr{G}_{3,2}=\left\{\left(x,-\left(-3-2 a-4 c+(2+2 a+2 c) x-a x^{2}-c x^{3}\right)\right) \mid a, c \in \mathbb{Q} c \neq 0, a \neq-1-2 c, x \text { root of } B_{3}(x)=0\right\}
$$

Therefore, we have the fourth family $\mathscr{G}_{3}=\mathscr{G}_{3,1} \cup \mathscr{G}_{3,2}$.

\section{Cases $m=4$ and $m=6$}

For $m=4$ : it exists a fonction $F$ such that $\operatorname{div}(F)=R_{1}+R_{2}+R_{3}+R_{4}+P_{1}-5 \infty$, hence $F \in \mathscr{L}(5 \infty)$,

$$
F(x, y)=u+v x+w x^{2}+d y \quad(d \neq 0) .
$$

We have $F\left(P_{1}\right)=0$, therefore $u+d=0$, then $F(x, y)=u+v x+w x^{2}-u y,(u \neq 0)$. At points $R_{i}$, we have $y=1+a x+c x^{2}$. Substiting $y$ to $y^{2}=x^{5}+1$, we have

$$
x\left(x^{4}+c^{2} x^{3}+2 a c x^{2}+\left(2 c+a^{2}\right) x+2 a\right)=0 .
$$

Simplifiying by $x$, we have the minimal polynomial

$$
B_{4}(x)=x^{4}+c^{2} x^{3}+2 a c x^{2}+\left(2 c+a^{2}\right) x+2 a=0 .
$$

We must have $a \neq 0$. We obtain a family of quartic points :

$$
\mathscr{G}_{4,1}=\left\{\left(x,+\left(1+a x+c x^{2}\right)\right) \mid a, c \in \mathbb{Q}, a \neq 0, x \text { root of } B_{4}(x)=0\right\} .
$$

For $m=6$ : by a similar argument as in previous case, we get a family of quartic points :

$$
\mathscr{G}_{4,2}=\left\{\left(x,-\left(1+a x+c x^{2}\right)\right) \mid a, c \in \mathbb{Q}, a \neq 0, x \text { root of } B_{4}(x)=0\right\}
$$

Therefore, we have the firth family : $\mathscr{G}_{4}=\mathscr{G}_{4,1} \cup \mathscr{G}_{4,2}$.

\section{Case $m=5$}

It exists $F$ such that $\operatorname{div}(F)=R_{1}+R_{2}+R_{3}+R_{4}+P_{0}-5 \infty$, so $F \in \mathscr{L}(5 \infty)$, then

$$
F(x, y)=u+v x+w x^{2}+d y \quad(d \neq 0) .
$$

We have $F\left(P_{0}\right)=0$, so $v=u+w$, therefore $F(x, y)=u+(u+w) x+w x^{2}+d y$. At points $R_{i}$, we have $y=-a+(-a-c) x-c x^{2}$ with $a=\frac{u}{d}$ and $c=\frac{w}{d}$. Substiting $y$ to $y^{2}=x^{5}+1$, we have

$$
(x+1)\left(x^{4}+\left(c^{2}+1\right) x^{3}+\left(c^{2}+2 a c-1\right) x^{2}+\left(2 a c+a^{2}+1\right) x+a^{2}-1\right)=0 .
$$

Simplifliying by $x+1$, we have the polynomial

$$
B_{5}(x)=x^{4}+\left(c^{2}+1\right) x^{3}+\left(c^{2}+2 a c-1\right) x^{2}+\left(2 a c+a^{2}+1\right) x+a^{2}-1 .
$$

We must have $a \neq \pm 1$, therefore, we have the fifth family :

$$
\mathscr{G}_{5}=\left\{\left(x,\left(-a+(-a-l) x-c x^{2}\right)\right) \mid a, c \in \mathbb{Q}, a \neq \pm 1, x \text { root of } B_{5}(x)=0\right\} .
$$




\section{References}

[1] N. Bruin, M. Stoll, The Mordell-Weil sieve : proving the nonexistence of Rational points on curves, LMS J. Comp. Math., 13 (2010), 272 -306.

[2] R. F. Coleman, Effective Chabauty Duke Math. J. 52(3) (1985), 765-770.

[3] E. F. Schaefer, Computing a Selmer group of a Jacobian using functions on the curve, Mathematische Annalen, 310 (1998), $447-471$.

[4] M. J. Klassen, E. F. Schaefer, Arithmetic and geometry of the curve $x^{4}=y^{3}+1$, Acta Arithmetica LXXIV.3 (1996) $241-257$.

[5] M. Fall, O. Sall, Ponts algébriques de petit degré sur la courbe d'équation affine $y^{2}=x^{5}+1$, Afr. Mat. 29 (2018) $1151-1157$.

[6] O. Sall, Points algébriques sur certains quotients de courbes de Fermat, C. R. Acad. Sci. Paris Sér I 336 (2003) $117-120$.

[7] S. Siksek, M. Stoll, Partial descent on hyper elliptic curves and the generalized Fermat equation $x^{3}+y^{4}+z^{5}=0$, Bulletin of the LMS 44 (2012) 151 $-166$ 